\title{
KALMAN FILTERING FOR THE EVALUATION OF THE CURRENT-TIME FUNCTION IN d.c. POLAROGRAPHY
}

\author{
M. Bos \\ Department of Chemical Technology, Technical University Twente, P.O. Box 217, 7500 AE Enschede,
} The Netherlands

(Received 4 November 1985. Accepted 21 February 1986)

\begin{abstract}
Summary-Kalman filtering was applied to the current vs. time data obtained at the growing mercury drop of a DME under d.c. polarographic conditions, to separate the faradaic and capacitive components of the electrode current. Polarograms consisting of the pure faradaic current vs. applied d.c. potential were subjected to a four-parameter curve-fitting procedure to obtain the polarographic characteristics, viz. half-wave potential, limiting current and slope of the log plot together with the baseline current. The method was tested with cadmium and zine in the $10^{-6}-10^{-5} M$ range. The standard deviations of the half-wave potentials and the limiting current/concentration ratios were found to be $1.0 \mathrm{mV}$ and 0.04 respectively.
\end{abstract}

In polarography the analytical signal from the faradaic process at the electrode is obscured at low concentrations by the capacitive response caused by the charging of the electrical double-layer capacitance. Many methods have been devised to minimize the contribution of this capacitive component to the compound current signal, mostly by instrumental techniques, e.g., tast and differential pulse polarography. With the advent of powerful on-line computers another approach has become viable in which mathematical processing of the measurements is used to separate the faradaic and capacitive components of the electrode current.

Numerous examples of the use of sophisticated data-processing techniques, such as the fast Fourier transform (FFT) procedure, curve-fitting, pattern recognition and Kalman filtering, combined with elaborate measurement procedures can be found in the literature for the a.c., pulse and stripping polarographic techniques. ${ }^{1-7}$ Direct-current polarography, however, is still a favourite technique for the study of complex-formation equilibria. ${ }^{810}$ It has the advantage that the processes at the electrode are well understood and described by relatively simple equations. ${ }^{11}$ In d.c. polarography the problem of separating the faradaic and capacitive response is mainly solved by mathematical data-processing. If this separation can be successfully achieved, a lower detection limit and better precision for the half-wave potential and limiting current can be obtained, owing to the absence of distortion of the polarographic wave by the capacitive component. This considerably extends the range of application of the polarographic method for determining stability constants. Moreover complicated schemes for baseline correction become superfluous, as the baseline will be a horizontal straight line. The feasibility of this approach has been demonstrated by Soong and Maloy, ${ }^{12}$ who applied a Rieman-Liouville transform (RLT) to the current-time data obtained at the growing mercury drop during normal d.c. polarography, to separate the faradaic and capacitive responses.

This paper describes an alternative route, the Kalman filter technique, to reach this goal. It has the advantage that a more complex model, with more than two parameters, can be accommodated and that it can directly process the incoming $i-t$ data without the need to gather and store the complete set of measurements which is characteristic for curve-fitting methods. The data-processing algorithm is rather simple and its speed allows real-time processing.

\section{THEORY}

\section{The instantaneous current in d.c. polarography}

The instantaneous cell current in d.c. polarography consists of a faradaic and a capacitive component:

$$
i(t)=i_{\mathrm{f}}(t)+i_{\mathrm{c}}(t)
$$

The capacitive part $i_{c}(t)$ can be described by

$$
i_{\mathrm{c}}(t)=k_{\mathrm{c}} t^{1 / 3}
$$

The constant $k_{\mathrm{c}}$ is potential-dependent, but can be considered constant during the growth of a given mercury drop if the voltage scan-rate is sufficiently low. For the instantaneous faradaic current the time-dependence is generally expressed by a $\frac{1}{6}$ order parabola, as in the Ilkovič equation. However, owing to the spherical shape of the mercury drop there is a difference in the shape and size of the diffusion space inside and outside it. This gives rise to two different situations, depending on whether the product of the electrode reaction forms an amalgam or is transported back into the solution. Kůta and Smoler ${ }^{13}$ give 
a complete account of this subject and use the following relationship, derived by Koutecky ${ }^{14}$ and Weber, ${ }^{15}$ to express the instantaneous faradaic current:

$$
\begin{aligned}
i=\frac{0.732 n F D^{1 / 2} \mathrm{~cm}^{2 / 3} t^{1 / 6} \lambda}{\left(\lambda+\sqrt{D_{\mathrm{ox}} / D_{\mathrm{red}}}\right)} \\
\times\left[1+\frac{3.96 D_{\mathrm{ox}}^{1 / 2} t^{1 / 6}(\lambda \pm 1)}{m^{1 / 3}\left(\lambda+\sqrt{D_{\mathrm{ox}} / D_{\mathrm{red}}}\right)}\right]
\end{aligned}
$$

In this equation $\lambda$ denotes the potential function

$$
\lambda=\exp \left[-\left(E-E_{0}\right) n F / R T\right]
$$

and the other symbols are those normally used in polarography. The two situations arise from the use of either the plus (no amalgam formation) or the minus sign (amalgam formation) in the second term in the brackets of the numerator of equation (3). On the basis of these equations Kưta and Smoler showed that the instantaneous faradaic current can be expressed as

$$
i_{\mathrm{f}}(t)=k_{\mathrm{f}} t^{\beta}
$$

where $\beta$ is given by

$$
\beta=\frac{1}{6}+0.661 D^{1 / 2} m^{-1 / 3} t^{1 / 6} \theta
$$

with

$$
\theta=(\lambda \pm 1) /\left(\lambda+\sqrt{D_{\text {ox }} / D_{\text {red }}}\right)
$$

In their work these authors determined $\beta$ for the faradaic curves for various cases and found that they could use a time-independent mean value that was significantly different from $\frac{1}{6}$ and changed with voltage in the polarographic wave for the case of amalgam formation. For the total cell current, equations (1), (2) and (5) give rise to:

$$
i(t)=k_{\mathrm{c}} t^{-1 / 3}+k_{\mathrm{f}} t^{\beta}
$$

This model equation (8) can be used in the Kalman filter scheme to estimate the parameters $k_{\mathrm{c}}, k_{\mathrm{f}}$ and $\beta$. If the $k_{\mathrm{f}}$ data obtained in this way are plotted against d.c. potential the resulting polarograms will show horizontal straight baselines and plateaus because the capacitive current component has been eliminated completely.

\section{Kalman filter theory}

The Kalman filter is a mathematical technique that can be used to estimate the state of a process in real-time from noise-corrupted measurements on that process. ${ }^{16-18}$ Here only the operational equations for the evaluation of the $i-t$ curves of d.c. polarography by means of equation (8) are given. The state of the process is completely characterized by the parameters $k_{\mathrm{v}}, k_{\mathrm{f}}$ and $\beta$ and is denoted by the vector $x$. As these parameters are considered to be time-invariant in the description of the $i-t$ curve for a growing single mercury drop, the state-to-state transition matrix is the unity matrix. The equation for the measurements in which the current is averaged over the interval from $t$ to $t+\Delta t$ follows from the integration of (8) over this interval:

$$
\begin{aligned}
\bar{t}(t \rightarrow t+\Delta t)=x_{1}\left[t^{-1 / 3}\right. & \left.+(\Delta t / 6) t^{-4 / 3}\right] \\
& +x_{2}\left[t^{x_{3}}+\left(\Delta t x_{3} / 2\right) t^{\left(x_{3}-1\right)}\right]
\end{aligned}
$$

The elements of the state-to-measurement transition vector $C$ can now be calculated:

$$
\begin{aligned}
C_{1}= & \delta i(t) / \delta x_{1}=t^{-1 / 3}-(\Delta t / 6) t^{-4 / 3} \\
C_{2}= & \delta i(t) / \delta x_{2}=t^{x_{3}}+\left(\Delta t x_{3} / 2\right) t^{\left(x_{3}-1\right)} \\
C_{3}= & \delta i(t) / \delta x_{3}=x_{2}\left[t^{x_{3}} \ln (t)\right. \\
& \left.+(\Delta t / 2 t) t^{x_{3}}+\left(\Delta t x_{3} / 2 t\right) \ln (t) t^{x_{3}}\right]
\end{aligned}
$$

The mathematical model for the current-time relationship at constant potential at a growing mercury drop can be written as

$$
\check{x}^{*}=\hat{x}+\mathbf{F} \hat{w}
$$

and

$$
\check{y}^{*}=\mathrm{C} \check{x}^{*}+n^{*}
$$

in which $x$ denotes the state vector of the process, consisting of the elements $\left[k_{\mathrm{c}}, k_{\mathrm{f}}, \beta\right]: \hat{x}$ is the estimated current state of the process, and $\ddot{x}^{*}$ stands for the predicted state of the process one step ahead in time. The product $F \hat{w}$ is the process noise contribution. Equation (12) gives the measurement prediction $y^{*}$ which is calculated from the predicted state of the process $\check{x}^{*}$ by the state-to-measurement transition matrix $\mathbf{C}$ (in this case a vector). Its elements $\left[C_{1}, C_{2}, C_{3}\right]$ are given by equation (10); $n_{y}^{*}$ is the noise associated with the measurements. The predicted co-variance of the state $\check{V}^{*}$ is given by:

$$
\check{V}^{*}=\hat{V}+\mathbf{F} w \mathbf{F}^{\mathbf{T}}
$$

where $\hat{V}$ is the estimate of the current co-variance matrix. $\mathbf{F}$ and $w$ are defined in equation (11) and $\mathbf{F}$ is the transpose of $\mathbf{F}^{\mathrm{T}}$. The prediction of the output signal co-variance $V_{y}^{*}$ follows from:

$$
\breve{V}_{y}^{*}=\mathrm{C} \breve{V}^{*} \mathrm{C}^{\mathrm{\top}}+N_{\mathrm{y}}
$$

in which $N_{y}$ is the measurement noise variance. With the results of (13) and (14) the Kalman gain matrix $\mathbf{K}^{*}$ can be calculated as:

$$
\mathbf{K}^{*}=\left(\check{V}^{*} \mathbf{C}^{\mathrm{T}}\right) / \check{V}_{y}^{*}
$$

With this Kalman gain, the calculated prediction of the measurement, and the actual value of the measurement $y_{m}^{*}$, a better estimate of the state vector can be calculated when this actual measurement value becomes available:

$$
\hat{x}^{*}=\check{x}^{*}+\mathbf{K}^{*}\left[y_{\mathrm{m}}^{*}-y^{*}\right]
$$

A new estimate of the error co-variance matrix of the state can be found from:

$$
\hat{V}^{*}=\left(\mathbf{I}-\mathbf{K}^{*} \mathbf{C}^{*}\right) \check{V}^{*}
$$

I being the identity matrix.

Cycling through equations (11)-(17) for each new 
measurement constitutes the Kalman filter procedure and provides improving estimates of the state of the process, viz. the wanted values of the capacitive and faradaic contributions to the total cell current, together with the exponent of the time-dependence of the faradaic part. If this exponent [ $\beta$ in equation (8)] is known and not dependent on the d.c. voltage the filter equations reduce to a one-measurement twostate system for which the Kalman gain matrices for the cell current $v s$. time series can be calculated off-line before the experiment is run. The real-time calculations are then reduced to the application of equations (11), (12) and (16) for each new measurement.

\section{EXPERIMENTAL}

\section{Chemicals}

Potassium chloride, cadmium sulphate (both Merck Suprapur) and zinc sulphate (Merck reagent grade) were used as received. The nitrogen used to remove oxygen from the samples in the polarographic cell was from HoekLoos (very pure grade). All solutions were prepared with demineralized water that had been filtered through Millipore Q2 filters.

\section{Apparatus}

A PAR model 174 polarograph was equipped with a Radiometer polarographic stand, type E64, DLT1 drop-life timer, and type 950-116 mercury capillary. The capillary characteristics for a $41-\mathrm{cm}$ mercury head were: natural drop-time $3.7 \mathrm{sec}$ and drop-rate $2.39 \mathrm{mg} / \mathrm{sec}$ (in $0.01 \mathrm{M} \mathrm{KCl}$ with open circuit).

The polarographic instrumentation was interfaced to a PDP11/10 minicomputer (Digital Equipment Corp.) through the analogue-to-digital (A/D) and digital-toanalogue $(\mathrm{D} / \mathrm{A})$ converters in a Laboratory Peripheral System (LPS, Digital Equipment Corp.). Both the A/D and $D / A$ converters provided 12 -bit resolution and voltage ranges of $\pm 5 \mathrm{~V}$. Timing was controlled by the LPSKW real-time clock option of the LPS.

For comparison purposes computerized d.c. tast polarography experiments were run on a Metrohm E506 polarograph equipped with an E505 polarographic stand and interfaced to a BASIS 108 microcomputer as described earlier. ${ }^{9}$ The characteristics of the DME used in these experiments were natural drop-time $5.1 \mathrm{sec}$ and drop-rate $1.09 \mathrm{mg} / \mathrm{sec}(0.01 \mathrm{M} \mathrm{KCl}$, open circuit, mercury head $65 \mathrm{~cm})$.

\section{Computer programs}

Two computer programs were written in FORTRAN IV. One was based on the one-measurement two-state description, in which the faradaic and capacitive factors $k_{\mathrm{f}}$ and $k_{\mathrm{c}}$ were estimated by the Kalman fiter for a given and fixed exponent $\beta$ for the time-dependence of the faradaic component of the current. In the second program this exponent $\beta$ was estimated along with $k_{\mathrm{f}}$ and $k_{\mathrm{c}}$. The data-acquisition routines were written in the assembly language MACRO-11 and linked to the main FORTRAN routine. The current measurements were performed at $80-\mathrm{msec}$ intervals. To eliminate line-frequency noise the current measurements were programmed in such a way that during each $80-\mathrm{msec}$ interval a window of one line-frequency period $(20 \mathrm{msec})$ was used, during which a series of current measurements was performed at a sampling frequency of $20 \mathrm{kHz}$. This series was then averaged and the result used as $y_{m}^{*}$ in the Kalman filter equations [ $f$. equation (16)]. Synchronization of these current measurements with the mercury drop-life was controlled by the electromechanical drop-life timer, which was activated from within the program.
For the one-measurement three-state filter the software is not fast enough to keep up with the 80 -msec interval between the measurements. In this case the current $v s$. time data have to be stored and the Kalman filter calculations performed after all measurements on a single mercury drop have been completed. Although this slows down the record. ing of a polarographic wave considerably, it offers the possibility of reiterating all data through the filter, making the procedure less sensitive to bad initial estimates for the states and the co-variance matrix. Moreover, a fair initial estimate for the states can be calculated by using the assumption that the capacitive component will have decayed completely for the last data-point. This will give a good initial estimate for the faradaic part which, in turn, can be applied to the first data-point to estimate the capacitive contribution. The initial estimate used for $\beta$ was 0.1667 .

The program output the $k_{\mathrm{f}} v s$. d.c. potential data to a chart recorder, which then displayed d.c. polarograms from which the capacitive-current contribution had been eliminated. These data were also stored in a disk file for use by a curve-fitting program that calculated half-wave potential, limiting $k_{f}$, slope of the log plot, and baseline current of the polarographic waves, from the $k_{\mathrm{f}} v s$. d.c. potential values. The program used was an extension of a previously published three-parameter procedure, ${ }^{19}$ the baseline current being the fourth parameter. This feature is especially useful when baseline extrapolation methods fail owing to the presence of a preceding polarographic wave in the vicinity of the wave of interest.

\section{Procedures}

The polarographic experiments were run with solutions that had been deoxygenated by passage of nitrogen for $15 \mathrm{~min}$. During the recording of the polarograms nitrogen was passed over the solution. The current/time data for the growing mercury drops were obtained by sampling the current at $80-\mathrm{msec}$ intervals. For cadmium 20 data-points were acquired from a single drop, whereas for zinc the current was sampled 40 times per drop. The current/time data were normally evaluated with the one-measurement two-state Kalman filter, with a fixed value for $\beta$. The value to use for $\beta$ was determined beforehand with the onemeasurement three-state procedure. In the calculation the variance of the measurements was set at one $A / D$ converter unit and the process noise was set to zero. The initial estimates for the diagonal co-variance matrix elements were set at unity, the off-diagonal elements at zero.

\section{RESULTS AND CONCLUSIONS}

Table 1 shows the results obtained with the nethod for cadmium in the concentration range $10^{-5}-10^{-6} M$. The supporting electrolyte was $0.01 M$ potassium chloride. The corresponding polarograms were calculated from the $i-t$ curves for the growing mercury drops at different d.c. potentials. The onemeasurement two-state $\left(k_{\mathrm{f}}\right.$ and $\left.k_{\mathrm{c}}\right)$ model was used in

Table 1. Kalman filter d.c. polarography for $\mathrm{Cd}(\mathrm{II}) /$ $0.01 \mathrm{M} \mathrm{KCl}$

\begin{tabular}{cccc}
\hline $\begin{array}{c}\text { [Cd], } \\
10^{-6} M\end{array}$ & $\begin{array}{c}\text { Limiting } k_{\mathrm{f}}, \\
n A\end{array}$ & $\begin{array}{c}E_{1 / 2}, \\
m V\end{array}$ & $\begin{array}{c}\text { Limiting } k_{\mathrm{f}} /[\mathrm{Cd}], \\
10^{6} n A .1 . \text { mole }^{-1}\end{array}$ \\
\hline 0.99 & 23.2 & -568.7 & 23.5 \\
1.96 & 49.9 & -568.8 & 25.5 \\
2.91 & 68.0 & -569.5 & 23.4 \\
3.85 & 92.7 & -569.4 & 24.1 \\
5.66 & 142.1 & -568.9 & 25.1 \\
Mean & & $-569.0 \pm 0.4$ & $24.3 \pm 0.9$ \\
\hline
\end{tabular}


Table 2. Kalman filter d.c. polarography for $\mathrm{Zn}(\mathrm{II})$ / $0.01 \mathrm{M} \mathrm{KCl}$

\begin{tabular}{rccc}
\hline $\begin{array}{c}\text { [Zn], } \\
10^{-6} M\end{array}$ & $\begin{array}{c}\text { Limiting } k_{\mathrm{f}}, \\
n A\end{array}$ & $\begin{array}{c}E_{1 / 2}, \\
m V\end{array}$ & $\begin{array}{c}\text { Limiting } k_{\mathrm{f}} /[\mathrm{Zn}], \\
10^{6} n A . l . \text { mole }^{-1}\end{array}$ \\
\hline 3.98 & 27.0 & -991.0 & 6.78 \\
9.90 & 66.8 & -991.8 & 6.75 \\
19.60 & 130.3 & -993.2 & 6.65 \\
29.10 & 188.2 & -990.7 & 6.47 \\
38.50 & 254.8 & -991.0 & 6.62 \\
Mean & & $-991.5 \pm 1.0$ & $6.65 \pm 0.12$ \\
\hline
\end{tabular}

the Kalman filter procedure. The average value for $\beta$ was found to be 0.19 from a preliminary experiment in which the one-measurement three-state $\left(k_{\mathrm{f}}, k_{\mathrm{c}}\right.$ and $\beta$ ) model was used. The same value was calculated by Küta and Smoler. ${ }^{13}$ The characteristics of the polarographic waves were evaluated with the fourparameter (limiting $k_{\mathrm{f}}, E_{\mathrm{l} / 2}$, slope and baseline current) curve-fitting procedure described above. From this table it can be concluded that d.c. polarographic characteristics can be obtained for cadmium with good constancy, down to a concentration of $10^{-6} \mathrm{M}$. Although more sensitive electroanalytical methods are available, such as anodic-stripping or squarewave polarography, ${ }^{20}$ the method described here has the advantage that its results can be used directly in well established methods that rely on d.c. polarographic data to calculate physicochemical parameters, e.g., complex-formation constants.

The results also indicate that it is possible to fit the baseline faradaic current as a fourth parameter along with the limiting $k_{\mathrm{f}}, E_{1 / 2}$ and slope of the log plot. There is then no need for baseline current extrapolation procedures, which means that systems that do not give a distinct baseline section in the polarogram can be evaluated by using only the rising part of the waves. Similar results to those obtained for cadmium were also found for the zinc system (Table 2). For this the average value of $\beta$ was found to be 0.27 . The one-measurement three-state procedure used here to determine the average value of $\beta$ can also be used for more fundamental studies of the nature of the electrode process. ${ }^{13}$ In the Kalman filter calculations the value of the integral double-layer capacitance is also determined as part of the process. In this work no attention was paid to these values, but clearly this facility makes the method a suitable tool for the study of double-layer phenomena. In particular the potential corresponding to zero charge can be obtained accurately and easily from a plot of the integral double-layer capacitance $v s$. d.c. potential.

For comparison d.c. tast experiments were run for zinc and cadmium. The lower concentration limit of which the results were of quality similar to those obtained with the Kalman filter technique turned out to be $10^{-5} \mathrm{M}$, as can be seen from Table 3 . The reason for the breakdown of the d.c. tast method below this
Table 3. Computerized d.c. tast polarography for $\mathrm{Zn}$ (II)/ $0.01 \mathrm{M} \mathrm{KCl}$

\begin{tabular}{rccc}
\hline $\begin{array}{c}{[\mathrm{Zn}],} \\
10^{-6} M\end{array}$ & $\begin{array}{c}\text { Limiting current, } \\
n \boldsymbol{A}\end{array}$ & $\begin{array}{c}E_{1 / 2}, \\
m \boldsymbol{V}\end{array}$ & $\begin{array}{c}i_{\mathrm{d}} /[\mathrm{Zn}], \\
n A . l . \text { mole }^{-1}\end{array}$ \\
\hline 5.74 & 11.4 & -983.3 & 1.99 \\
7.15 & 16.9 & -984.6 & 2.36 \\
8.55 & 21.6 & -985.5 & 2.52 \\
11.31 & 33.5 & -985.0 & 2.96 \\
31.7 & 117.4 & -986.5 & 3.70 \\
49.7 & 196.6 & -986.7 & 3.96 \\
66.3 & 269.1 & -986.9 & 4.06 \\
83.3 & 336.5 & -987.0 & 4.03 \\
97.4 & 396.7 & -986.0 & 4.07 \\
\hline
\end{tabular}

concentration is mainly the uncertainty introduced by the baseline extrapolation needed. Owing to the substantial contribution of the capacitive component when high sensitivity is used for current measurement, the curvature of the baseline changes with d.c. potential in a complex manner, thus ruling out simple baseline-correction techniques for d.c. tast polarography at high sensitivities.

Acknowledgements - The author wishes to thank Prof. W. E. van der Linden for his general support, Mrs. A. M. W. van Veen Blaauw for doing the d.c. tast experiments, Ir. A. R. van Heusden for his help with Kalman filter theory and Mrs. B. E. M. M. Verbeeten for preparing the manuscript.

\section{REFERENCES}

1. D. E. Smith, Anal. Chem., 1976, 48, 517A.

2. R. J. O'Halloran, J. C. Schaar and D. E. Smith, ibid., 1978, 50, 1073.

3. P. F. Seelig and R. de Levie, ibid, 1980, 52, 1506

4. P. F. Seelig and H. N. Blount, ibid., 1976, 48, 252.

5. T. F. Brown, D. M. Caster and S. D. Brown, ibid, 1984, 56, 1214.

6. J. E. Anderson and A. M. Bond, ibid., 1983, 55, 1934.

7. M. Ichise, H. Yamagishi and T. Kojima, J. Electroanal. Chem. Interfacial Electrochem., 1978, 94, 187.

8. A. Laouenan and E. Suet, Talanta, 1985, 32, 245.

9. D. Ph. Zollinger, M. Bos, A. M. W. van Veen-Blaauw and W. E. van der Linden, Anal. Chim. Acta, 1985, 167, 89.

10. J. A. Santaballa, C. Blanco, F. Arce and J. Casado, Talanta, 1985, 32, 931.

11. D. J. Leggett, ibid., 1980, 27, 787.

12. F. Soong and J. T. Maloy, J. Electroanal. Chem. Interfacial Electrochem., 1983, 153, 29; J. T. Maloy and F. Soong, Proc. Intern. Symp. LCEC and Voltammetry, Indianapolis, 1983, pp. 102-106.

13. J. Kůta and I. Smoler in Progress in Polarography, P. Zuman and I. M. Kolthoff (eds.) Vol. I, p. 43. Interscience, New York, 1962.

14. J. Koutecky, Czech. J. Phys, 1953, 2, 51 .

15. J. Weber, Coll. Czech. Chem. Commun., 1959, 24, 1424.

16. R. E. Kalman, Trans. A.S.M.R., Ser. D, J. Basic Eng., $1960,82,35$.

17. R. E. Kalman and R. S. Bucy, ibid., 1961, 83, 95.

18. A. H. Jazwinski, Stochastic Processes and Linear Filtering Theory, Chapters 7, 8, 9. Academic Press, New York, 1970.

19. M. Bos, Anal. Chim. Acta, 1976, 81, 21 .

20. J. J. O'Dea, J. Osteryoung and R. A. Osteryoung, $J$. Phys. Chem., 1983, 87, 3911. 